\title{
Theoretical model atmosphere spectra used for the calibration of infrared instruments
}

\author{
L. Decin ${ }^{1,2, \star}$ and K. Eriksson ${ }^{3}$ \\ ${ }^{1}$ Department of Physics and Astronomy, Institute for Astronomy, K. U. Leuven, Celestijnenlaan 200 D, 3001 Leuven, Belgium \\ e-mail: Leen.Decin@ster.kuleuven.ac.be \\ 2 Sterrenkundig Instituut Anton Pannekoek, University of Amsterdam, Kruislaan 403, 1098 Amsterdam, The Netherlands \\ 3 Department of Astronomy and Space Physics, Uppsala University, Box 515, 75120 Uppsala, Sweden
}

Received 12 March 2007 / Accepted 7 June 2007

ABSTRACT

Context. One of the key ingredients in establishing the relation between input signal and output flux from a spectrometer is accurate determination of the spectrophotometric calibration. In the case of spectrometers onboard satellites, the accuracy of this part of the calibration pedigree is ultimately linked to the accuracy of the set of reference spectral energy distributions (SEDs) that the spectrophotometric calibration is built on.

Aims. In this paper, we deal with the spectrophotometric calibration of infrared (IR) spectrometers onboard satellites in the 2 to $200 \mu \mathrm{m}$ wavelength range. We aim at comparing the different reference SEDs used for the IR spectrophotometric calibration. The emphasis is on the reference SEDs of stellar standards with spectral type later than A0, with special focus on the theoretical model atmosphere spectra.

Methods. Using the MARCS model atmosphere code, spectral reference SEDs were constructed for a set of IR stellar standards (A dwarfs, solar analogs, G9-M0 giants). A detailed error analysis was performed to estimate proper uncertainties on the predicted flux values.

Results. It is shown that the uncertainty on the predicted fluxes can be as high as $10 \%$, but in case high-resolution observational optical or near-IR data are available, and IR excess can be excluded, the uncertainty on medium-resolution SEDs can be reduced to $1-2 \%$ in the near-IR, to $\sim 3 \%$ in the mid-IR, and to $~ 5 \%$ in the far-IR. Moreover, it is argued that theoretical stellar atmosphere spectra are at the moment the best representations for the IR fluxes of cool stellar standards.

Conclusions. When aiming at a determination of the spectrophotometric calibration of IR spectrometers better than 3\%, effort should be put into constructing an appropriate set of stellar reference SEDs based on theoretical atmosphere spectra for some 15 standard stars with spectral types between A0 V and M0 III.

Key words. instrumentation: spectrographs - techniques: spectroscopic - stars: atmospheres - stars: late-type - infrared: stars techniques: photometric

\section{Introduction}

For (IR) spectrometers, three main steps have to be covered to establish the relation between input signal, flux, and wavelength: spatial, spectral, and photometric calibration. In this paper, we focus on the reference spectral energy distributions (SEDs) used in the spectrophotometric $(=$ spectral+photometric) calibration process, with main emphasis on the spectral calibration part.

For (IR) spectrometers onboard satellites, the determination of the relative spectral response function (RSRF), which characterises the wavelength-dependent response of a spectrometer, is often a two-step process: (1) first, the RSRF is determined during laboratory tests from measuring a cryogenic blackbody calibration source at differing temperatures; (2) after launch, the RSRF is refined by comparing observations with reference SEDs of various celestial calibration sources, preferably at a spectral resolution comparable to that of the instrument.

Whenever an instrument covers a new spectral window or has a higher sensitivity than preceding instruments, new reference SEDs have to be constructed. This was and will be the case for instruments onboard the ESA-Infrared Space Observatory (ISO), the NASA-Spitzer satellite, the ESA-Herschel mission, the NASA-James Webb Space Telescope (JWST), and many

\footnotetext{
* Postdoctoral Fellow of the Fund for Scientific Research, Flanders.
}

others. In this paper, emphasis will be put on the reference SEDs of calibration sources for instruments covering the 2 to $200 \mu \mathrm{m}$ wavelength range. When selecting calibration sources, issues like brightness in the wavelength regime covered by the instrument, confusion by neighbouring sources, sky visibility, and pointing accuracy are to be considered. In the $2-200 \mu \mathrm{m}$ wavelength range, planets, asteroids, and cool standard stars are the ideal calibration sources to cover the whole dynamic range of present-day instruments (see Sect. 2). This paper focuses on the reference SEDs of standard stellar candles, with the aim of assessing the reliability and achieving accuracy when using IR stellar standards for spectral calibration purposes. Quite often, calibration scientists or observers (have to) rely on "quoted" errors, without having insight into this part of the calibration pedigree. Since for a proper interpretation of the output signal one needs to have a grip on the uncertainties that propagate to the fluxes ultimately calculated by an instrument, the discussion in this paper is required.

Section 2 gives an overview of the different IR flux calibration sources. The status of stellar reference SEDs in the IR is discussed in Sect. 3, and we focus on the theoretical atmosphere spectra in Sect. 4. Different stellar reference SEDs are compared in Sect. 5, and we end with the conclusions in Sect. 7. 


\section{Infrared flux calibration sources}

In this section, we discuss different IR calibration sources used in the spectrophotometric calibration process.

\subsection{Planets}

For calibration purposes at wavelengths longer than $\sim 30 \mu \mathrm{m}$, planets are extremely useful since they are among the few astronomical objects bright enough in the far-IR to allow sufficiently accurate flux density predictions. At $100 \mu \mathrm{m}$, the flux values for Neptune and Uranus range between 100 and $1000 \mathrm{Jy}$. Uranus is often used as the primary calibrator, as it is known to be a reliable calibrator (Griffin \& Orton 1993; Sidher et al. 2003), while Neptune and Mars are used as secondary calibrators.

Good models for Uranus exist (e.g., Moreno 1998; Orton \& Burgdorf 2003; Sidher et al. 2003). Radiative transfer calculations are done in spherical geometry and take the limb into account. The models do not, however, take chemical reactions into account, nor heating and cooling. In the $\mathrm{mm}$ and submmregimes, the models are confirmed within the accuracy of 10$20 \%$. The big advantage of Mars over Uranus is that it is bright. Different models for Mars' surface exist (e.g. Rudy et al. 1987; Lellouch et al. 2000; Hartogh et al. 2005). Using Mars as a primary calibrator, however, would pose different problems: (1) Mars is a planet with a surface and an atmosphere, both contributing to the continuum emission; (2) sandstorms influence the line shapes; (3) the ice caps can influence the continuum if the ice is melting. Neptune could serve almost equally well as an independent (secondary) calibrator. However, many large features are visible in the Voyager IRIS spectra, which may indicate systematic variability with rotational phase (Bishop et al. 1998).

\subsection{Asteroids}

Typical surface temperatures of main-belt asteroids are such that they emit the bulk of their thermal radiation in the far-IR. At wavelengths longer than $\sim 20 \mu \mathrm{m}$, the largest asteroids are brighter than the brightest IR stellar sources: they cover the flux range between 100 and $1000 \mathrm{Jy}$ in the wavelength range between 30 and $45 \mu \mathrm{m}$, and hence fill a gap where stellar calibrators are not available (see Fig. 1 in Müller \& Lagerros 1998).

The work of Müller (Müller \& Lagerros 1998, 2002, 2003; Müller et al. 2005) has largely improved the accuracy of the theoretical model SEDs of asteroids. The most accurate SEDs at present are based on the thermophysical model (TPM) for describing the asteroids' thermal emission (Müller \& Lagerros 1998). Due to the asteroids' orbit, brightness temperatures vary by $17 \pm 13 \%$. Müller \& Lagerros (2002) quote an accuracy of $5 \%$ between 5 and $200 \mu \mathrm{m}$ for Ceres, Pallas, and Vesta, i.e. for the three largest asteroids.

The main limitations for asteroids as IR calibration sources come from the changing background conditions and the flux changes on timescales of hours due to rotation. Moreover, the list of reliable asteroids is still short.

\subsection{Stars}

In the near-IR, stars are ideal calibrators, especially since they are almost point-like and span a range in flux level more than 4 orders of magnitude between 2 and $50 \mu \mathrm{m}$, and one can create a database covering the whole sky. Stellar standards are, however, quite faint in the far-IR and can henceforth not be used as primary calibrators at the far-IR wavelength ranges. Good
IR calibration sources need to comply several criteria: be single and non-variable, not have an IR excess due to a chromosphere, debris disk, or a circumstellar envelope, and be located in an uncrowded region that can be observed all or most of the time (Decin et al. 2007). Preferably, the IR standard is cooler than $\sim 10000 \mathrm{~K}$ to provide a good signal-to-noise ratio over most of the wavelength range.

In the case of spectrophotometric calibration, one extra requirement should be added to this list regarding the composition of the sample of used calibrators with regard to the spectral types. For photometric calibration purposes, the use of only one spectral type can be justified (Diaz-Miller 2007), but in the case of a spectrometric calibration, where every single wavelength needs to be calibrated, none of the available reference SEDs of any spectral type has high enough accuracy in the full 2 to $200 \mu \mathrm{m}$ wavelength range (see Sect. 3). Three classes of spectral stellar standards have been commonly used in the IR spectrometric calibration pedigree: (i) early A dwarfs, (ii) solar analogs, and (iii) late-type giants, usually of spectral type G9-M0 III. Each of these groups provides a different challenge (see below), but by combining them, they $(i)$ reduce the chances for systematic errors, possibly introduced by the use of only one spectral type, and (ii) they will increase the total accuracy since the best SED can be used for each part of the spectrum.

\section{Status of stellar reference SEDs in the IR}

\subsection{Black body}

Planck curves represent the simplest way to model stellar farIR fluxes. A comparison with the predictions of a solar continuum model shows clear deviations, arising to more than $20 \%$ in the far-IR (see Fig. 14 in van der Bliek et al. 1996). The reason is the contribution of the $\mathrm{H}^{-}$free-free opacity, shifting the flux-forming region more to outer cooler layers. Neither line or continuous absorption nor back-warming effects are/can be included.

\subsection{Engelke function}

The Engelke function (Engelke 1992) is a more sophisticated two-parameter analytical approximation to the $2-60 \mu \mathrm{m}$ infrared continuum spectrum for giants and dwarfs with effective temperature, $T_{\text {eff }}$, between 3500 and $6000 \mathrm{~K}$. This spectral function is based on the scaling of a semi-empirical (plane-parallel) solar atmospheric profile to differing effective temperatures. The result describes the continuum spectrum expected for stars in terms of their effective temperature and angular size. The estimated probable error in absolute flux values is quoted to be $\sim 3 \%$ below $10 \mu \mathrm{m}$, growing to $\sim 5 \%$ around $25 \mu \mathrm{m}$ and $6 \%$ at $60 \mu \mathrm{m}$.

The main limitation of the Engelke function is neglect of the influence of the surface gravity. In Fig. 1 the difference between the Engelke function and the continuum flux of a plane-parallel theoretical stellar atmosphere (see Sect. 4) with an effective temperature, $T_{\text {eff }}$, of $3500 \mathrm{~K}$ for different values of the gravity is shown. For values of the logarithm of the gravity, $\log g$, lower than 3.00, the absolute deviations may be as high as $25 \%$. At a temperature of $6000 \mathrm{~K}$, the absolute deviations approximate the errors quoted by Engelke (1992). Important, however, for the discussion in this paper of the RSRF determination are the systematic differences between the Engelke continuum and both (1) the continua calculated by ab-initio model atmosphere calculations (see Fig. 1) and (2) real continua deduced from observations (see Engelke et al. 2006). This systematic discrepancy 


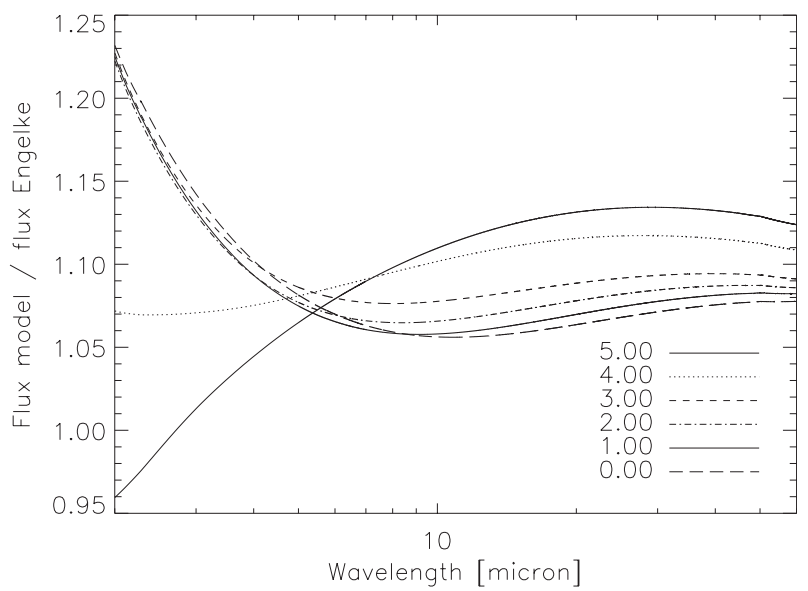

Fig. 1. Ratio between the continuum flux predicted from a theoretical plane-parallel stellar atmosphere with $T_{\text {eff }}=3500 \mathrm{~K}$ and values for the logarithm of the gravity ranging between 5.00 and 0.00 and the flux computed from the Engelke function.

occurs both for solar analogs and the cooler K-M giants: the same type of curves as displayed for the lower gravity values in Fig. 1 (M-giants) also occur in an analogous plot for $\mathrm{G}$ dwarfs, but with a smaller amplitude of $\sim 3 \%$. Note also that for almost all values of $\log g$, the ratio as displayed in Fig. 1 is systematically higher than one, implying that when either the effective temperature or the angular diameter $\left(\theta_{\mathrm{d}}\right)$ is kept fixed, the other parameter will be overestimated.

Another issue not taken into account are sphericity effects, important when dealing with the extended atmospheres of giants. Figure 2 compares a theoretical atmosphere spectrum (see Sect. 4) calculated using spherical geometry with the spectrum obtained in plane-parallel geometry for the case of an M0 III giant. Compared to a plane-parallel atmosphere, the radiation field of a spherical model is diluted in the upper photospheric layers, causing the temperature (and hence the source function) there to be lower. The lower surface flux is, however, compensated by the fact that the IR flux arises from higher layers. The net result is an infrared excess of the spherical model relative to the plane-parallel model. Important for this paper is the rising continuum in the bottom panel of Fig. 2, implying that the use of the Engelke function or a plane-parallel geometry to represent the SED of giants may introduce uncertainties on the broad-band RSRF characterisation of a few percent.

\subsection{The IR calibration framework by Cohen et al.}

In a series of papers on spectral irradiance calibration in the infrared, Cohen and colleagues have presented a self-consistent network of absolutely calibrated reference spectra between 1.2 and $35 \mu \mathrm{m}$ for over 600 stars spanning spectral types from A0 to M4 and distributed over the entire sky (Cohen et al. 1992a,b, 1995, 1996a, 1999, 2003, often referred to as the CWW network). The CWW network is the result of a tremendous and consistent work done during the past 15 years. Four main steps can be distinguished in the network. (i) The approach is based on a pair of absolutely calibrated models of the two A-type dwarfs Vega and Sirius calculated by Kurucz (1993) (Cohen et al. 1992a). (ii) The next layer in the network is a set of composites of 13 secondary calibration stars. Composites are spectra measured by various ground-based and airborne telescopes (KAO, IRAS LRS, etc.) that are averaged and/or spliced together to form a continuous spectrum between 1.2 and $35 \mu \mathrm{m}$. This set

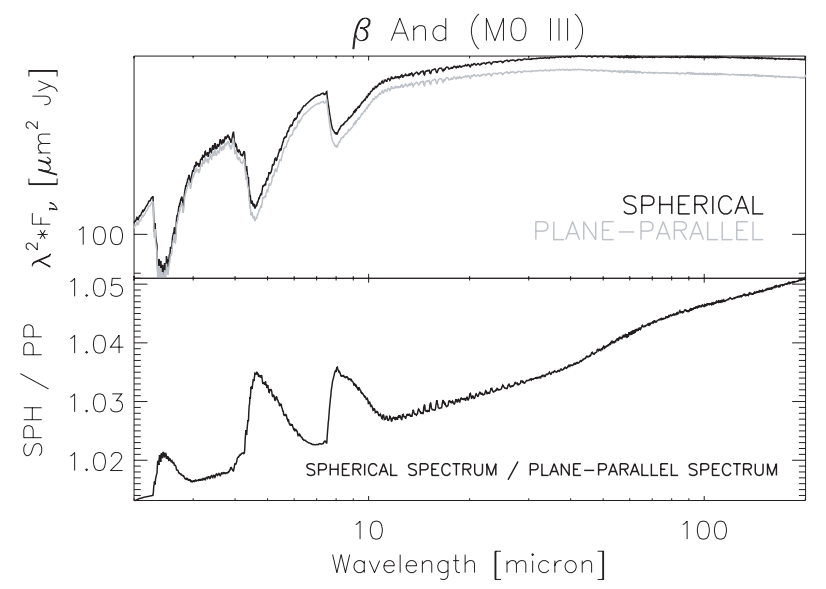

Fig. 2. Upper panel: model atmosphere spectrum in spherical geometry (black) compared to the theoretical spectrum calculated in planeparallel geometry (grey) representing the M0 III giant $\beta$ And $(\lambda / \Delta \lambda=$ 100). Bottom panel: ratio between spherical and plane-parallel spectrum. Stellar parameters are taken from Decin et al. (2003a).

compromises one G2 dwarf $-\alpha^{1}$ Cen (G2 V); seven K giants $-\alpha$ Tau (K5 III), $\alpha$ Boo (K2 IIIp), $\beta$ Gem(K0 III), $\alpha$ Hya (K3 II-III), $\alpha$ TrA (K2 III), $\epsilon$ Car (K3 III), $\gamma$ Dra (K5 III); and 5 M giants $-\beta$ Peg (M2.5 II-III), $\beta$ And (M0 III), $\alpha$ Cet (M1.5 III), $\gamma$ Cru (M3.4 III), $\mu$ UMa (M0 III) (Cohen et al. 1992b, 1995, 1996a,b). The KAO was no longer operational at the time the composites of $\gamma$ Dra, $\alpha$ Cet, $\gamma$ Cru and $\mu$ UMa were constructed. Spectral regions that are opaque from the ground were therefore replaced by spectra of other stars with the same spectral type. Note that the "composite" of $\alpha^{1}$ Cen is a Kurucz model-atmosphere spectrum, which was then used as a reference to create the composite spectra of $\alpha \operatorname{TrA}$ and $\epsilon$ Car. For wavelengths longer than $\sim 22 \mu \mathrm{m}$, the Engelke function was used for twelve composites to represent the stellar spectrum, the exception being $\alpha$ Tau. (iii) These 13 composite spectra are the basis for a set of absolutely calibrated spectral templates for 602 stars with spectral types between G9.5-M0.5 III (Cohen et al. 1999, 2003). For each star a smoothed composite spectrum is chosen as its spectral template according to the spectral class. The template is corrected for reddening and normalised using available optical photometry. For purpose of the NASA-Spitzer Infrared Array Camera (IRAC) calibration an extra set of absolutely calibrated $0.275-35 \mu \mathrm{m}$ spectra of 33 optical standard stars with spectral types between A0-A5 V and K0-M0 III, and flux levels down to $V \sim 11-12$, was prepared (Cohen et al. 2003). (iv) In support of the Infrared Space Observatory (ISO) PHT and LWS instruments, CWW have provided extrapolated continuum spectra of few composites till $300 \mu \mathrm{m}$ (Cohen et al. 1996b). The continuum spectra have been interpolated in a grid of four plane-parallel modelatmosphere spectra (see also Sect. 3.2) with solar abundances. To obtain the temperature-versus-continuum optical depth relation $\left(\tau_{\text {cont }}\right)$, a scaling based on the ratio $T_{\text {eff }}($ desired $) / T_{\text {eff }}$ (grid model) was performed. Differences in gravity were not accounted for. An uncertainty of $5.67 \%$ was assessed to these extrapolations.

It should be emphasised that the CWW network has some major advantages, the main ones being (1) the common calibration pedigree; and (2) the good sky-coverage (see Fig. 2 in Cohen et al. 1999). For that reason, the CWW network was used to support the calibration of many spaceborne, airborne, and ground-based instruments, as e.g. instruments onboard ISO, Spitzer, AKARI, MSX, etc. 
However, aiming for a (spectrophotometric) calibration better than a few percent, some steps used in the construction of the CWW network deserve some comments.

- A first issue concerns the danger of propagating of systematic errors from the top of the calibration pedigree. In this context, we want to note that the adopted spectra for the fundamental reference stars in the calibration pedigree are absolutely-calibrated Kurucz models of the A-type stars Sirius and Vega. Since no absolute flux measurements were available for Sirius, Cohen et al. (1992a) chose to calibrate the Sirius model absolutely with respect to Vega by using the observed magnitude differences in $K, L, L^{\prime}, M$, and at four mid-IR wavelengths. However, the use of Vega (or its model spectrum representing an "ideal" Vega without flux excess) is strongly debated (for an overview, we refer to Gray 2007). We summarise that (i) it is obvious that the Vega debris disk system contributes to the flux excess from this star beyond $10 \mu \mathrm{m}$ (e.g. Neugebauer et al. 1984); (ii) that recent results by Absil et al. (2006) show an excess of $1.29 \pm 0.19 \%$ in the $K$-band close-in to Vega (within $1^{\prime \prime}$ ) presumably due to a hot inner circumstellar disk; (iii) and Vega is a rapidly rotating pole-on star with a significant temperature gradient $(\sim 1500-$ $2250 \mathrm{~K}$ ) from its pole to its equator (Gulliver et al. 1994; Aufdenberg et al. 2006), so its SED differs from conventional models, not assuming a rotating star (Gray 2007). As noted by Bohlin (2007) a single-temperature $T_{\text {eff }}=9400 \mathrm{~K} \mathrm{Kurucz}$ model fits the Hubble Space Telescope (HST) STIS SED to $1-2 \%$ from 3200 to $10000 \AA$. However, a multi-temperature model may be required to achieve a $\sim 1 \%$ precision for shorter and longer wavelengths (the topic of this study). Finally (iv) previous work has suggested an inconsistency between infrared and visible measurements of Vega, when fitted to a standard A0 V star model (e.g. Rieke et al. 1985; Megessier 1995; Verhoelst 2005; Rieke et al. 2007), probably having its roots in Vega being a rapidly rotating star seen pole-on. When the absolute calibration of the Sirius spectrum by magnitudedifference with Vega is done in the optical and not in the IR, the fluxes of the Sirius spectrum increases by $\sim 3.7 \%$ (Verhoelst 2005; Rieke et al. 2007) compared to the absolute calibration as proposed by Cohen et al. (1992a), shifting the angular diameter of Sirius from 6.04 mas (Cohen et al. 1992a) to 6.15 mas (see Fig. 3). Based on the Midcourse Space Experiment (MSX) data, Price et al. (2004) propose an increase in the SED of Sirius by $1 \%$. This increase in the flux density of the primary standard Sirius has a direct influence on the absolute flux level of all composites and templates, whose absolute photometric data are based on relative flux differences compared to the primary standards.

- One should be aware that, for all composite spectra, with the exception of $\alpha$ Tau, the flux values between $\sim 22$ and $35 \mu \mathrm{m}$ are represented by the Engelke function. As discussed in the previous section, the neglect of both gravity and sphericity effects results in a systematic broad-band inaccuracy of $\sim 3 \%$ for early A dwarfs, rising to $\sim 8 \%$ for late $K$-giants. Cohen et al. (1996b) and Cohen (2007) justify this assumption by comparing the UKIRT CGS3 16-24 $\mu$ m spectra of $\alpha$ Boo and $\alpha$ Cet with their relevant Engelke approximation (see Fig. 5 in Cohen et al. 1996b) and from the KAO spectrum of $\alpha$ Tau (Cohen et al. 1992b). A comparison to these observations in the $\lambda^{4} F_{\lambda}$-space (Fig. 4) does, however, not give any more support to the "flatness" of an Engelke spectrum than to the rising continuum of a theoretical model atmosphere (see also Sect. 5). One can only argue that, from a physical

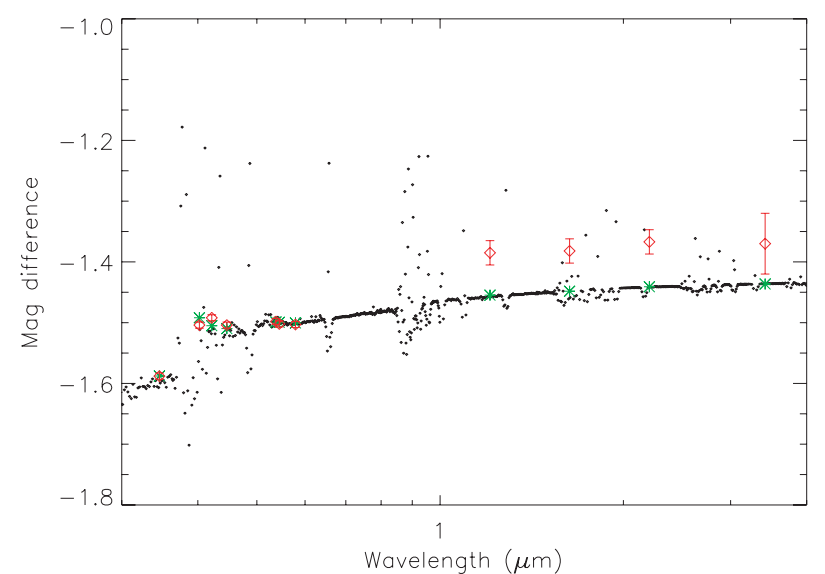

Fig. 3. Observed (red - diamonds) versus synthetic (green - stars) magnitude difference between Vega and Sirius $\left(\operatorname{Mag}_{\text {Vega }}-\operatorname{Mag}_{\text {Sirius }}\right)$ in the Geneva photometric system and the JHKL bands (Verhoelst 2005). Black dotted line represents the difference between both of Kurucz's models used in Cohen et al. (1992a), with stellar parameters $T_{\text {eff }}=9550 \mathrm{~K}, \log g=3.95,[\mathrm{Fe} / \mathrm{H}]=-0.3$ for Vega and $T_{\text {eff }}=9850 \mathrm{~K}$, $\log g=4.25,[\mathrm{Fe} / \mathrm{H}]=+0.5$ for Sirius. However, the Kurucz's model for Sirius is scaled in such a way as to match the observed difference in the Geneva photometric system, yielding an angular diameter for Sirius of 6.15 mas (instead of 6.04 mas as used in Cohen et al. 1992a). Clearly, the $(\mathrm{N}) I R$ magnitude differences are not compatible asthey differ by up $10 \%$.

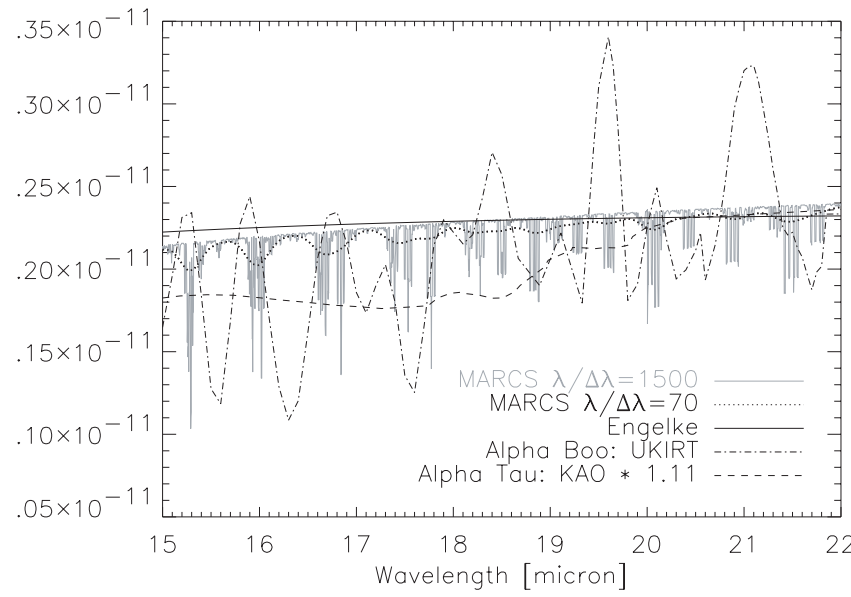

Fig. 4. UKIRT CGS3 15-22 $\mu \mathrm{m}$ spectrum for $\alpha$ Boo (K2 IIIp; unscaled, dash-dot line) and KAO spectrum for $\alpha$ Tau (K5 III; scaled by a factor 1.11, dashed line) are compared with the Engelke approximation (full line) with parameters as used by Cohen et al. (1996b) for $\alpha$ Boo $\left(T_{\text {eff }}=4362 \mathrm{~K}, \theta_{\mathrm{d}}=21.12 \mathrm{mas}\right)$, and an appropriate theoretical MARCS model at a resolution $\lambda / \Delta \lambda=1500$ (grey line) and at a resolution of $\lambda / \Delta \lambda=70$ (dotted line; corresponding to the UKIRT CGS3 resolution), with stellar parameters as determined in Decin et al. (2003a).

point of view, the increase in the $\mathrm{H}^{-}$opacity towards longer wavelengths $\left(\sigma\left(\mathrm{H}_{\mathrm{ff}}^{-}\right) \propto \lambda^{2}\right)$ yields a shift of the flux-forming region towards higher, cooler, atmospheric layers, and hence a decrease in the stellar surface flux.

- The assumption in the creation of a "template" spectrum is that a de-reddened spectrum of a star is representative of the infrared spectral signature of any star of the same two-dimensional spectral classification. This is only true to some extent. Differences in stellar parameters and photospheric abundances can result in substantial differences in the strength of molecular absorption bands as can be seen in Fig. 8. A more elaborated version of this figure can be found 
in Decin et al. (2000). For G-type stars and cooler, differences in stellar parameters within one spectral sub-class (e.g. from K1 III to K2 III) may cause differences in the (broad) NIR molecular absorption bands in the order of 5-8\% (at a resolution of 100), an uncertainty that should be directly translated to the uncertainties attributed to the template spectra. For hotter stars, the deviations are smaller and are mainly seen in the strength of the hydrogen lines. The continuum flux may differ by up to $\sim 4 \%$.

- Recently, Engelke et al. (2006) found, for a subset of 33 standards with spectral types between G2 and M5, available in the CWW network, that the derived spectra (by Engelke and co-workers) display systematic differences with the composites/templates in the CWW network, in that Engelke standard spectra have $4-7 \%$ lower flux in the $1-4 \mu \mathrm{m}$ spectral region. The spectra of Engelke et al. (2006) remove the A$\mathrm{K}$ star calibration bias recently noted in the calibration of Spitzer/IRAC, which was based on templates in the CWW network (Reach et al. 2005).

The net result is that the CWW network has provided the astronomical community with a consistent network containing some 600 stars. Calibration experiments for the SPIRIT III infrared instrument onboard the MSX have demonstrated that in the 6 (broad) spectral bands (with wavelength between 4.29 and $21.34 \mu \mathrm{m}$ ) the absolute flux calibration for 8 composites in the CWW network is $<2.3 \%$ (Price et al. 2004). However, previous discussion also shows that for some spectral regions of the IR SED, the uncertainties attributed to the template spectra or extrapolated continuum spectra in the CWW network should be larger than now quoted (for an overview, we refer to Table 1). A typical example of the attributed total uncertainty for two template spectra is given in Fig. 5. In the spectral regions where molecules absorb photons substantially, at least an $8 \%$ uncertainty (see above) should be attributed to the templated spectra. Another example is the (unknown) contribution of a (possible) chromosphere, ionised wind or dust-driven wind, which may yield uncertainties in the order of $10 \%$ at $100 \mu \mathrm{m}$ (see Sect. 4.3; van der Bliek et al. 1996), much higher than the 5.67\% uncertainty attributed to the extrapolated composite spectra by Cohen et al. (1996b).

In order to remove the "molecular feature" bias that can enter into the template construction, the computation of ab-initio theoretical model atmosphere spectra may offer a solution (see next section). Moreover, theoretical atmosphere spectra can be computed at any spectral resolution (comparable to that of the instrument) and in that way compete with the template spectra, which have quite low spectral resolution.

\subsection{Model atmosphere spectra}

Different groups have given a lot of effort into developing trustworthy hydrostatic 1-dimensional (1D) model atmosphere codes, the most famous ones in the field of cool stellar atmospheres $\left(T_{\text {eff }} \lesssim 10000 \mathrm{~K}\right)$ being the ATLAS atmosphere code (Kurucz 1970, 1993, 1996; Castelli \& Kurucz 2004), the MARCS code (originally presented in Gustafsson et al. 1975, with the current grid being described in Gustafsson et al. 2003), and the PHOENIX code (Hauschildt et al. 1999a,b). Also in the case of late-type (variable) asymptotic giant branch (AGB) stars, the 1D hydrostatic models are still useful in providing us with physical information, due to the great detail now included in their treatment of opacities. Hydrodynamic models in 1D (e.g. Bessell et al. 1996; Winters et al. 2000; Höfner et al. 2003) include
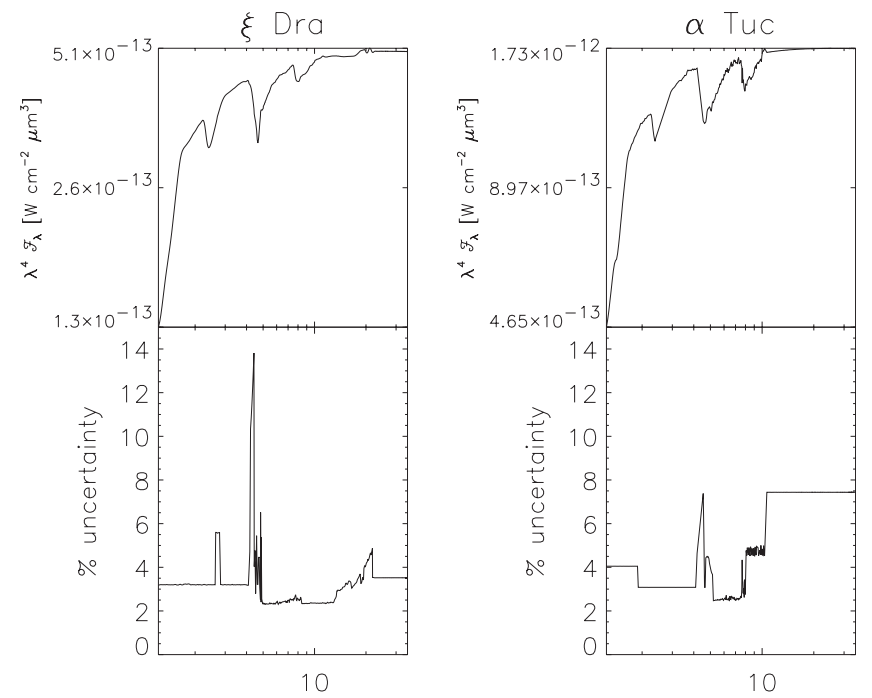

Fig. 5. Upper panels: Template spectra of $\xi$ Dra (K2 III) and $\alpha$ Tuc (K3 III). The composite spectrum of $\alpha$ Boo (K2 IIIp) has been used to construct the template of $\xi$ Dra (K2 III), and for $\alpha$ Tuc (K3 III) the composite of $\alpha$ Hya (K2 II-III) is used. Lower panels: the total uncertainty attributed to the templated spectra by Cohen et al. (1999). Main absorption features are the $\mathrm{CO}$ fundamental around $4.5 \mu \mathrm{m}$, $\mathrm{CO}$ first overtone around $2.3 \mu \mathrm{m}, \mathrm{SiO}$ fundamental around $8 \mu \mathrm{m}$ and $\mathrm{SiO}$ first overtone around $4.2 \mu \mathrm{m}$.

detailed physics, like time-dependent dust formation and the coupling between gas, dust, and radiation. Few dynamical models are now able to simultaneously solve the equations of hydrodynamcics and frequency-dependent radiative transfer leading to consistent dynamical density-temperature structures (see, e.g., Höfner et al. 2003). Using these kinds of models, one can already quantitatively reproduce the variation in line profiles due to the influence of gas velocities (see, e.g., Nowotny et al. 2005). Recently, 3D hydrodynamical simulations of stellar surface convection have become feasible thanks to advances in computer technology and efficient numerical algorithms (Collet et al. 2006). The 3D models can shed light on the coupling between convection and pulsation, as well as be employed in element abundance analysis. These 3D model computations are, however, still very computer-time intensive, and it is not known how well they represent the surface layers of the stars, so spectrum synthesis of a whole sample of stellar spectra is still beyond the scope.

For the scope of this project of developing accurate SEDs for IR stellar standards, we therefore rely on a 1D hydrostatic model atmosphere code. We here opt to use the MARCS code (see next section).

\section{Status and accuracy of MARCs model atmosphere spectra in the IR}

The MARCs model atmosphere and spectrum synthesis code was developed in Uppsala (Gustafsson et al. 2003) ${ }^{1}$. This local thermodynamic equilibrium (LTE) model atmosphere code is built on the assumptions of spherical or plane-parallel stratification in homogeneous stationary layers and hydrostatic equilibrium. Energy conservation is required for radiative and convective flux, where the energy transport due to convection was treated through a local mixing-length theory. For a discussion of

\footnotetext{
1 http://marcs.astro.uu.se
} 
Table 1. Summary of uncertainties attributed to the standard star spectra in the CWW network in the 2-200 $\mu \mathrm{m}$ range.

\begin{tabular}{llll}
\hline \hline Description & Uncertainty & Spectral type & Wavelength region \\
\hline $\begin{array}{l}\text { - propagation of systematic errors } \\
\text { e.g. due to use of Vega as prime calibrator }\end{array}$ & & & \\
$\rightarrow$ absolute flux calibration of Sirius w.r.t. Vega & $1-3 \%$ & A-M & $2-200 \mu \mathrm{m}$ \\
- use of Engelke function (Engelke 1992) & $3 \%$ & A-dwarfs & $22-35 \mu \mathrm{m}$ \\
& $8 \%$ & K-giants & $22-35 \mu \mathrm{m}$ \\
- creation of "template" spectrum & up to 8\% & G-K & around 2.3,4.0,4.2, $8 \mu \mathrm{m}$ \\
$\rightarrow$ molecular features & up to 4\% & A-M & $2-200 \mu \mathrm{m}$ \\
$\rightarrow$ continuum & $210 \%$ & G-M & $\lambda>100 \mu \mathrm{m}$ \\
- presence of chromosphere/ionised wind & $\gtrsim 10 \%$ & A-M & $\lambda>2 \mu \mathrm{m}$ \\
presence of circumstellar dust & $4-7 \%$ & G2-M5 & $1-4 \mu \mathrm{m}$ \\
- systematic difference with new Engelke & & & \\
$\quad$ standards (Engelke et al. 2006) & &
\end{tabular}

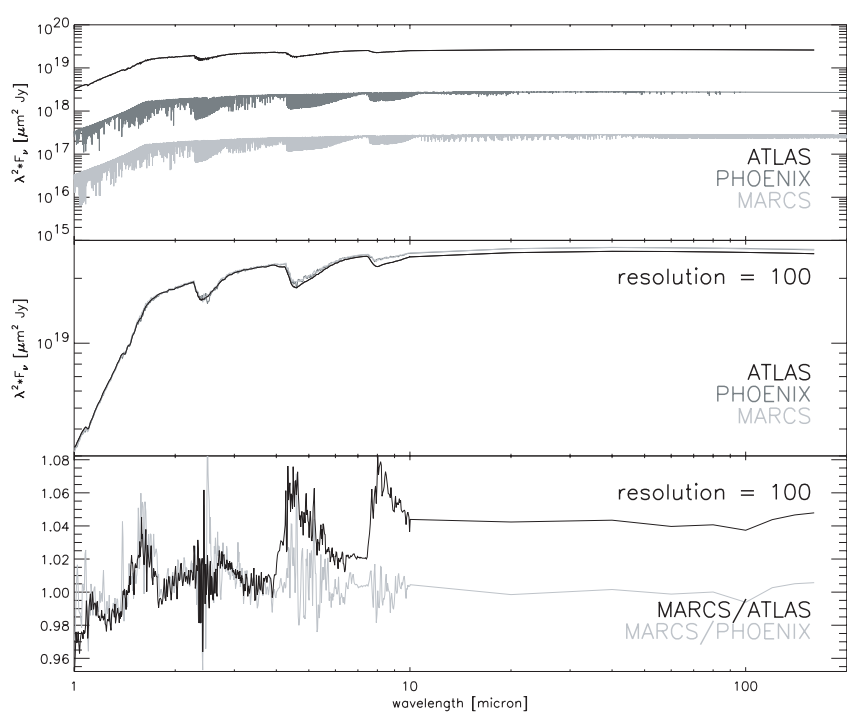

Fig. 6. Upper panel: comparison between the ATLAS, PHOENIX, and MARCS model spectra. The PHOENIX model is shifted downwards with a factor 10, and the MARCS model with a factor 100. Middle panel: comparison between the ATLAS, PHOENIX and MARCS model spectra at a resolution of 100 for $\lambda \leq 10 \mu \mathrm{m}$. At longer wavelengths, only the 8 wavelength points as given in the ATLAS models (at 20, 40, 60, 80, $100,120,140$, and $160 \mu \mathrm{m}$ ) are retained. Difference between PHOENIX and MARCS model fluxes is barely visible. Bottom panel: ratio between MARCS and ATLAS (black) and PHOENIX (gray) model fluxes.

the method for solving the radiative transfer equations in the atmospheric models and spectrum synthesis, we refer to Nordlund (1984) and Plez et al. (1992).

In the framework of the release of a grid of MARCS models, colleagues from the University of Uppsala are comparing the model structures of the MARCS, ATLAS, and PHOENIX codes. An example typical of a cool $\mathrm{K}$ giant is shown in Fig. 6. The plot is made in the $\lambda^{2} F_{v}$-space to clearly distinguish the differences. The continuum level between the MARCS and PHOENIX models do agree within $0.5 \%$. A larger difference of $\sim 5 \%$ is seen between the MARCS and ATLAS models for the continuum at $\lambda>10 \mu \mathrm{m}$. The difference in the molecular features is $\$ 4 \%$ between MARCS and PHOENIX and $\$ 8 \%$ between MARCS and ATLAS at a resolution of 100 . The reason for the difference is the use of a plane-parallel geometry in the case of the ATLAS models, whereas the PHOENIX and MARCS models use a spherically symmetric geometry. Slightly different surface temperature and geometrical extension explain the difference between MARCS and PHOENIX model fluxes. Moreover, the MARCS models are based on the Asplund et al. (2004) solar abundances, while the PHOENIX models follow the results of Grevesse et al. (1996). Note also that the MARCS model uses a more complete $\mathrm{OH}$ and $\mathrm{SiO}$ line list at $\lambda>10 \mu \mathrm{m}$ (see also Fig. 7).

For spectrum synthesis, data on the absorption by atomic and molecular species are collected from different databases. For a discussion of various available atomic and molecular line lists, we refer to Decin (2000). For the purpose of this work, the following infrared spectroscopic line lists were used: CO line list computed by Goorvitch \& Chackerian (1994), SiO by Langhoff \& Bauschlicher (1993), CN by Plez (priv. comm.), OH by Goldman et al. (1998), $\mathrm{H}_{2} \mathrm{O}$ by Partridge \& Schwenke (1997), $\mathrm{NO}, \mathrm{HF}, \mathrm{NH}, \mathrm{HCl}$, and $\mathrm{CH}$ by Sauval (priv. comm.), and atomic line lists by van Hoof (1998), by Hirata \& Horaguchi (1995), by Sauval (priv. comm.), and of VALD (Piskunov et al. 1995; Ryabchikova et al. 1997; Kupka et al. 1999). Using the model photosphere as input, synthetic spectra are calculated for a typical resolution of $\lambda / \Delta \lambda \sim 300000$, even though the final instrumental resolution is often lower. With a typical microturbulence of $2 \mathrm{~km} \mathrm{~s}^{-1}$, this means we are certain to sample all lines in the atomic and molecular database. We note that, for the purpose of calculating theoretical spectra in the mid to far-IR, some line lists are still far from complete or accurate (see Sect. 4.5). An typical example of a $\mathrm{K} 2$ giant is shown in Fig. 7. At a medium resolution of $\lambda / \Delta \lambda=1500$, the depression of flux due to the line veiling is $\sim 3 \%$ at wavelengths longer than $\sim 30 \mu \mathrm{m}$.

For the purpose of using model atmosphere spectra to represent the SED of standard stars, an appropriate analysis of different sources of uncertainties contributing to total uncertainty in the spectrum predictions is in place. The following sections discuss the effects of sources of error as $a$ ) the input stellar parameters, $b$ ) uncertainties in the model assumptions, $c$ ) the possible presence of a chromosphere, ionised wind or circumstellar dust shell, $d$ ) the adopted continuum opacity, and $e$ ) the used line lists.

\subsection{Dependency on stellar parameters}

Model atmospheres are defined by the fundamental parameters effective temperature, gravity, and metallicity (and stellar mass or radius in case of a spherical geometry). As demonstrated in Decin et al. (2000), the influence of the stellar mass on the synthetic spectrum is small. Varying the other fundamental 


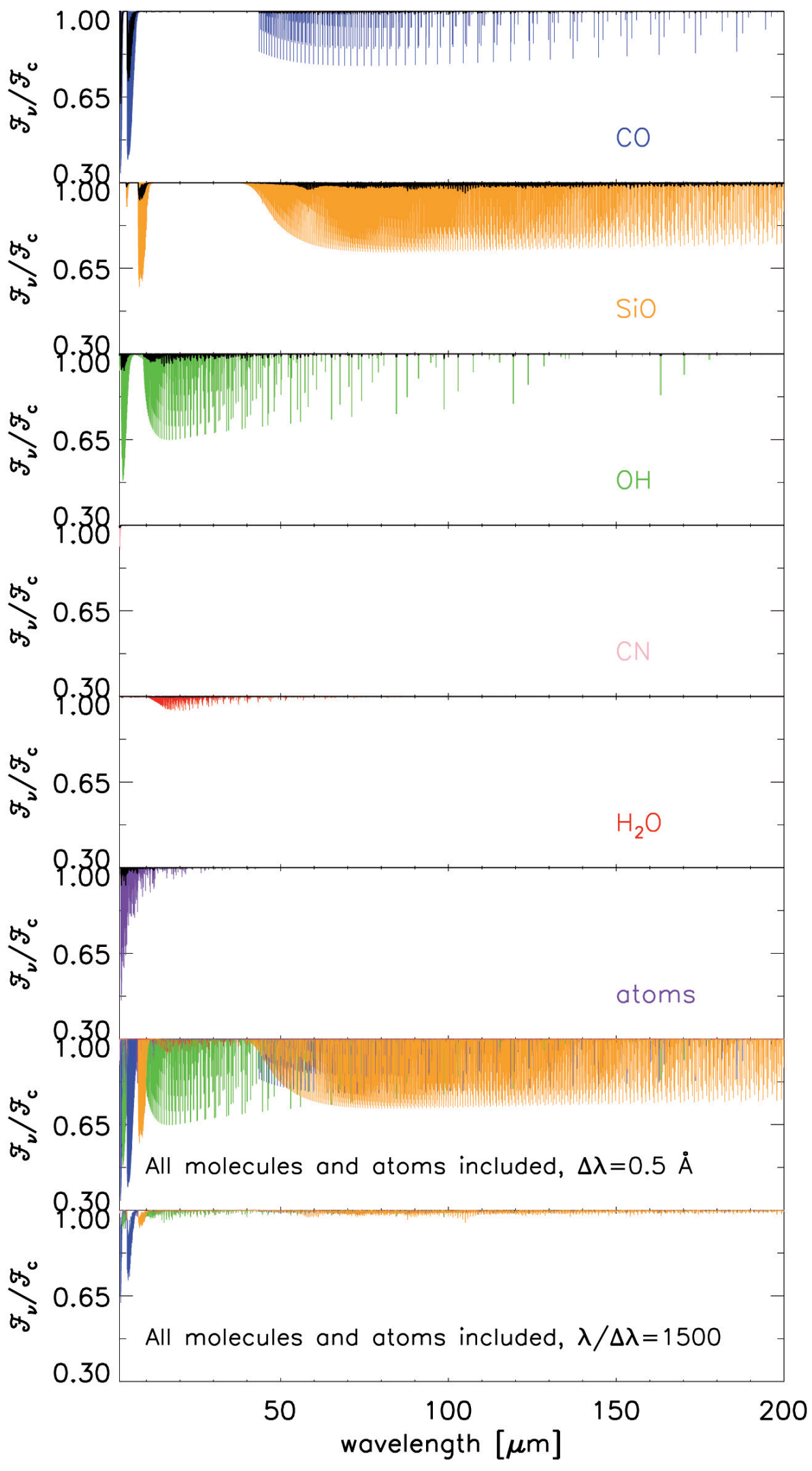

Fig. 7. Contribution of $\mathrm{CO}$ (blue), $\mathrm{SiO}$ (orange), $\mathrm{OH}$ (green), $\mathrm{CN}$ (pink), $\mathrm{H}_{2} \mathrm{O}$ (red), atoms (purple) to the 2-200 $\mu \mathrm{m}$ synthetic spectrum calculated from the atmosphere model with stellar parameters $T_{\text {eff }}=4320 \mathrm{~K}, \log g\left[\mathrm{~cm} / \mathrm{s}^{2}\right]=1.50$, mass $M=1.1 M_{\odot},[\mathrm{Fe} / \mathrm{H}]=-0.50$, microturbulence $\xi_{t}=2 \mathrm{~km} \mathrm{~s}^{-1}, \varepsilon(\mathrm{C})=7.96, \varepsilon(\mathrm{N})=7.61$, $\varepsilon(\mathrm{O})=8.68, \varepsilon(\mathrm{Mg})=7.33, \varepsilon(\mathrm{Si})=7.20$, and ${ }^{12} \mathrm{C} /{ }^{13} \mathrm{C}=7$. The first six panels display the contribution at a resolution $\Delta \lambda=0.5 \AA$ in color and at a medium resolution $\lambda / \Delta \lambda=1500$ in black. The total contribution to the full spectrum is shown in the seventh panel at the high resolution of $\Delta \lambda=0.5 \AA$, and in the bottom panel at the medium resolution of $\lambda / \Delta \lambda=1500$. Note the lack of atomic absorption features for wavelengths longer than $\sim 50 \mu \mathrm{m}$. parameters shows the effect of errors in the determination of the fundamental parameters on the synthetic flux distribution. Although this effect is dependent on the full set of fundamental stellar parameters, we may summarise that, for $\lambda>50 \mu \mathrm{m}$ (1.), varying the effective temperature by $\sim 200 \mathrm{~K}$ for stars with spectral type between $\mathrm{G}$ and $\mathrm{K}$, roughly corresponds to a change of $\sim 4 \%$ in the continuum flux in the IR, (2.) a change in the logarithm of the surface gravity of 0.20 dex introduces uncertainties in the continuous flux distribution of about $0.5 \%$, and (3.) an uncertainty in the metallicity of about 0.20 dex corresponds to an uncertainty in the IR continuum flux of $\sim 0.1 \%$. The uncertainty in the near-IR and on molecular absorption features is, however, 


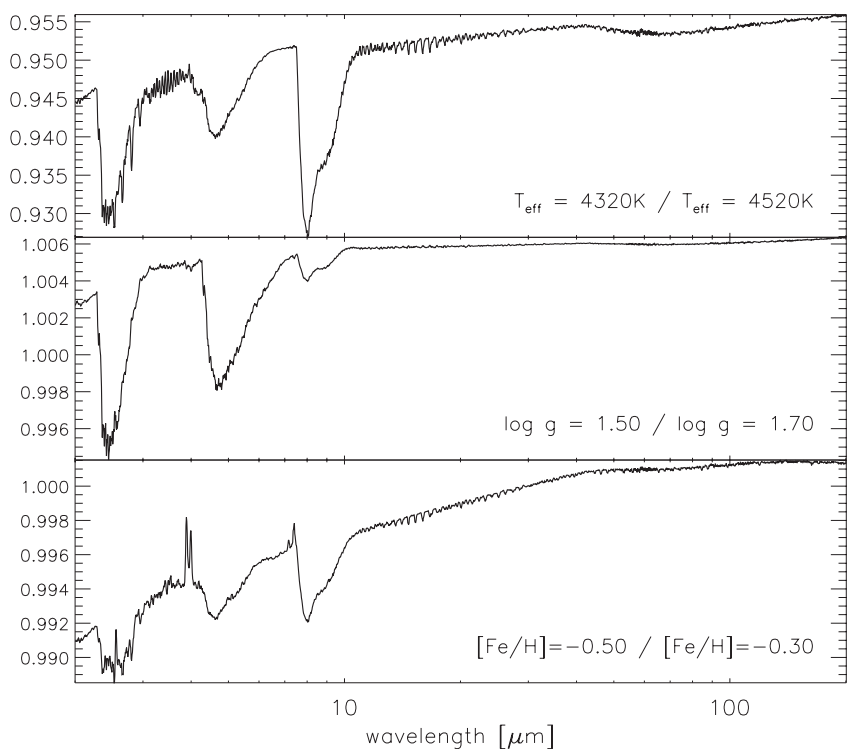

Fig. 8. Ratio between synthetic spectra with differing stellar parameters at a resolution of $\lambda / \Delta \lambda=100$. The standard model has the same stellar parameters as in Fig. 7. Upper panel: ratio between a synthetic spectrum with $T_{\text {eff }}=4320 \mathrm{~K}$ and with $T_{\text {eff }}=4520 \mathrm{~K}$. Middle panel: ratio between $\log g=1.50$ and $\log g=1.70$. Bottom panel: ratio between $[\mathrm{Fe} / \mathrm{H}]=-0.50$ and $[\mathrm{Fe} / \mathrm{H}]=-0.30$.

much greater, as can easily be seen in Fig. 8, arising to 7\% (at a resolution of 100)

The same order of magnitude on the uncertainties also applies to the continuum predictions of the hotter A-type stars for the same relative changes in stellar parameters. On one hand, one may argue that the absence of broad molecular absorption bands makes these hotter dwarfs more eligible as standards. On the other hand, exactly the presence of molecular features in the cooler giants can be used as a strong diagnostic tool to estimate the stellar parameters, and hence to provide a fiducial prediction of the whole IR spectrum. Especially in spectral regions with strong hydrogen lines, for which the computation of the selfbroadening remains problematic (Barklem et al. 2000; Decin et al. 2003b), the use of cooler giant as stellar standards will improve the accuracy of the spectrophotometric calibration.

\subsection{The temperature distribution $T(\tau)$}

Model atmosphere calculations are based on a number of assumptions, one of them being radiative and convective flux conservation also in the outermost layers of the photosphere. This simplification may be the reason that the, otherwise almost perfect, match between the FTS-Kitt Peak Spectrum of $\alpha$ Boo and theoretical predictions based on MARCS atmospheres (see Figs. 16-17 in Decin et al. 2003b) shows small deviations at the $1-2 \%$ level at a resolution of $\lambda / \Delta \lambda \sim 60000$ in the lowexcitation $\mathrm{CO}$ and $\mathrm{OH}$ lines.

Not only for K giants (as $\alpha$ Boo), but also for the most studied star, the Sun, there is still quite some debate concerning the assumed mean thermal profile in the outer layers of the photosphere. Both for $\mathrm{G}$ and $\mathrm{K}$-type stars, indications are found from, e.g., the $\mathrm{Ca}$ II $\mathrm{H}$ and $\mathrm{K}$ lines that the temperature structure has a minimum before segueing into the mechanically heated chromosphere (e.g. Ayres \& Linsky 1975). Controversially, the analysis of CO lines indicates a cooler brightness temperature at the same altitude (e.g. Ayres \& Testerman 1981; Wiedemann et al. 1994; Ayres et al. 2006).
As done by van der Bliek et al. (1996) and Vanhollebeke (2003), one can simulate uncertainties in the temperature structure $T(\tau)$ to mimic different effects: $a$ ) flatten or steepen $T(\tau)$ for a Rosseland optical depth $\tau_{\text {ross }}<0.01$ to study the effect of less, respectively more, line blanketing; $b$ ) steepen $T(\tau)$ around $\tau_{\text {ross }}=0.1$ to study the effect of convective overshoot in the outer layers of the model photosphere; or $c$ ) steepen $T(\tau)$ for $\tau_{\text {ross }}>1$ to study the effect of convection in the deeper layers of the atmosphere. From Table 3 in van der Bliek et al. (1996) and the results of Vanhollebeke (2003), we can conclude that this type of error in the temperature distribution gives rise to uncertainties in the predicted continuum flux at $100 \mu \mathrm{m}$ lower than $3.5 \%$. This uncertainty can, however, be strongly reduced in case highresolution spectra are available for atomic or molecular lines with different strengths and excitation energies. From a proper analysis of the line shapes, one can pin down $T(\tau)$ to $\sim 50 \mathrm{~K}$ in the outer layers, reducing the uncertainties in IR continuиm flux predictions to $\sim 1-2 \%$ (van der Bliek et al. 1996).

\subsection{The presence of a chromosphere, ionised wind, or circumstellar dust shell}

One of the largest uncertainties in model spectrum predictions is the (unknown) presence of a chromosphere, an ionised wind, or a circumstellar dust envelope. These structures may easily yield a flux excess in the order of $10 \%$ at mid to far-infrared wavelengths (van der Bliek et al. 1996). Recent studies by Dehaes et al. (2007) show that all of the six studied cool standard stars, used for the calibration of the Short-Wavelength Spectrometer (SWS, 2.38-45.2 $\mu \mathrm{m}$ ) onboard ISO, with spectral types between K2 III and M0 III show a flux excess due to an ionized wind. Only for two of them, the flux excess starts at wavelengths shorter than $1 \mathrm{~mm}$, but still longer than $\sim 200 \mu \mathrm{m}$. In the case of the Spitzer-IRS $(5.3-18 \mu \mathrm{m})$ calibration, the IRS team had to reject a considerable fraction of candidate standards as calibrators due to IR excess emission (Sloan 2005, priv. comm.).

\subsection{The continuous opacity source: $H_{f f}^{-}$}

The continuous opacity of solar-type and cooler stars is dominated by free-free absorption of the negative hydrogen ion. The MARCS model atmosphere calculations make use of the freefree absorption coefficients as calculated by Bell \& Berrington (1987). A quantitative assessment of the reliability of $\mathrm{H}_{\mathrm{ff}}^{-}$absorption coefficients by John (1994) shows that the absorption coefficients tabulated by Bell \& Berrington (1987) are accurate to about $1 \%$ for wavelengths greater than $0.5 \mu \mathrm{m}$ over the temperature range between 1400 and 10080 K. Stilley \& Callaway (1970) neglected the adiabatic exchange potential term yielding absorption coefficients that may be off by $\sim 4.5 \%$ at temperatures lower than $\sim 2500 \mathrm{~K}$. Using these coefficients, one may introduce errors in the order of $4 \%$ at wavelengths $<10 \mu \mathrm{m}$, diminishing to less than $0.3 \%$ for longer wavelengths (see Fig. 9). The prominent feature, seen around $8 \mu \mathrm{m}$ in Fig. 9, arises from the response of $\mathrm{SiO}$ on the slightly different temperature structure.

\subsection{Line lists}

Atomic and molecular data bases (including very different numbers of molecular lines and based on computations spanning a wide range in quality) are used in the construction of model atmospheres. Knowing the limitations on the accuracy and completeness of the used line list is of key importance when 
Table 2. Summary of uncertainties attributed to the theoretical atmosphere spectra in the $2-200 \mu \mathrm{m}$ range at a resolution $\lambda / \Delta \lambda \sim 100$.

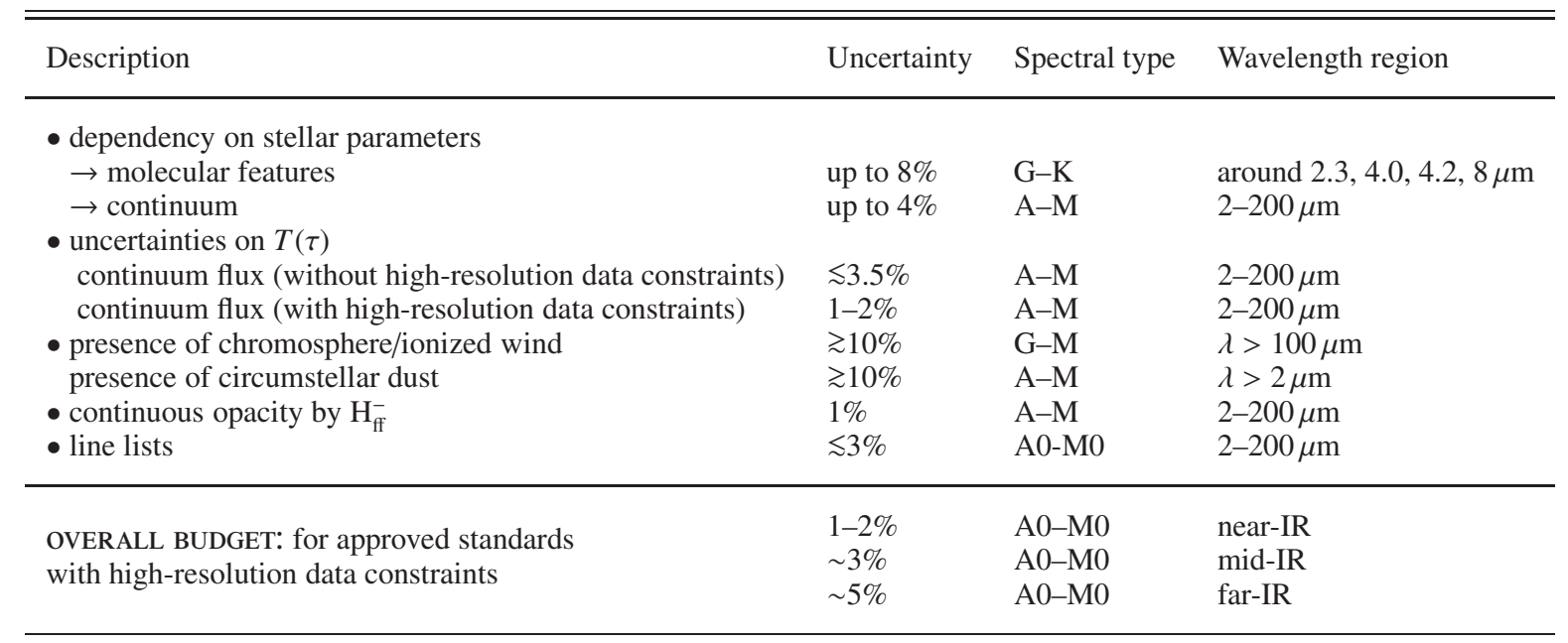

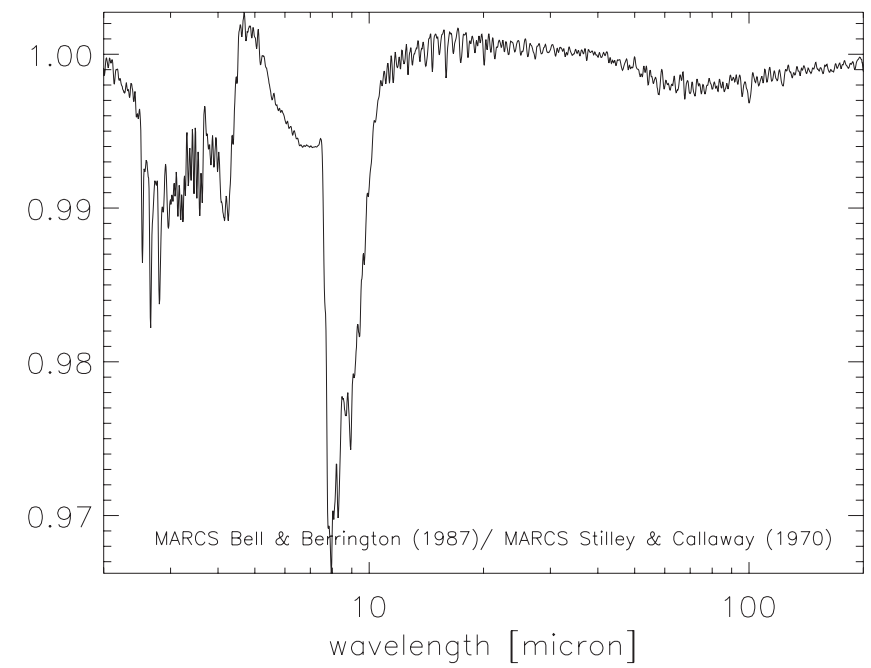

Fig. 9. Ratio between the synthetic spectrum calculated with the $\mathrm{H}_{\mathrm{ff}}^{-}$ absorption coefficients of Bell \& Berrington (1987) and the spectrum based on the $\mathrm{H}_{\mathrm{ff}}^{-}$absorption coefficients of Stilley \& Callaway (1970). The same stellar parameters as in Fig. 7 are used, data are reduced to a resolution of $\lambda / \Delta \lambda=100$.

calculating theoretical spectra. The MARcs code, with the input data used, is particularly tuned for computations of late-type stars where molecular opacities play an important role. As said in the introduction of Sect. 4, we here rely on the study done by Decin (2000). In this study, it has been shown that for molecules such as $\mathrm{CO}, \mathrm{SiO}$, and $\mathrm{OH}$, different molecular line lists reach a high level of accuracy and completeness. In the case of $\mathrm{CN}$, the dissociation energy is, however, still a matter of debate (see, e.g., Lambert 1994). Most problematic is the situation for the $\mathrm{H}_{2} \mathrm{O}$ line lists. The most often used theoretical $\mathrm{H}_{2} \mathrm{O}$ data bases are the SCAN list (Jørgensen et al. 2001), the list of Partridge \& Schwenke (1997), and the very recent list of Barber et al. (2006). These line lists are likely to be satisfactory for opacity calculations and probably for comparing observed and synthetic spectra at low and medium resolution, but at higher resolution line positions based on laboratory measurements (e.g., from Polyansky et al. 1997) should be used if possible.

Focusing on the wavelength range between 60 and $210 \mu \mathrm{m}$ (covered by the ESA PACS instrument onboard Herschel, launch foreseen in 2008), one should realise that the atomic VALD ${ }^{2}$ database presently only contains 1380 lines with $\lambda<122 \mu$ m, the NIST database ${ }^{3}$ only 90 atomic lines, while the atomic line list of van Hoof (1998) tabulates 13527 lines, of which the oscillator strengths are only known for 1660 .

MARCS model atmospheres were used by, among others, Decin et al. (2000) and Ryde \& Eriksson (2002) to predict IR spectra of cool stars. In their comparison between the highresolution $(\lambda / \Delta \lambda \sim 60000)$ Fourier Transform Spectrometer (FTS) spectrum of $\alpha$ Boo and theoretical MARCS predictions, Decin et al. (2003b) show that, in the case of this well-known giant, the model predictions in the $0.9-5.3 \mu \mathrm{m}$ range only differ from the observational data by $1-2 \%$ ! In general, IR model spectra predictions in the wavelength range $<25 \mu \mathrm{m}$ for A-G dwarfs and for non-pulsating $\mathrm{K}-\mathrm{M} 0$ giants may be as accurate as $3 \%$ at medium resolution $(\lambda / \Delta \lambda \sim 1500)$ and as $\sim 5 \%$ at high resolution $(\lambda / \Delta \lambda \sim 100000)$, as judged from the available observational data (Decin et al. 2003b,a). An exception may be the hydrogenline predictions due to the problematic computation of the selfbroadening (Barklem et al. 2000). At longer wavelengths, the accuracy and resolution of today's modern instruments remain too poor to constrain the model atmosphere spectra at a few percent level. Luckily, the depression of flux due to the line veiling in the FIR is estimated to be $\$ 3 \%$ at a resolution of 1500 (see Fig. 7), rendering the representation of the SED of stellar standards by theoretical model atmosphere spectra still useful. In order to avoid the propagation of inaccurate predictions of molecular/atomic lines to the RSRF determination, different spectral classes should be used. At temperatures lower than $3500 \mathrm{~K}, \mathrm{H}_{2} \mathrm{O}$ becomes a dominant opacity source, excluding giant stars with a cooler spectral type than M0 III as standard calibrators.

\subsection{Summary}

In last few sub-sections many uncertainties on the calculations of model atmospheres are summarised (see Table 2 for an overview), and the total uncertainty is not (as often done) estimated as the root-sum-square of all above-mentioned uncertainties since they are often mutually dependent. The total uncertainty on the stellar reference SEDs depends on the spectral type of the target, the wavelength range under study and the

\footnotetext{
2 http://ams.astro.univie.ac.at/vald/

3 http://physics.nist.gov/cgi-bin/AtData/main_asd
} 


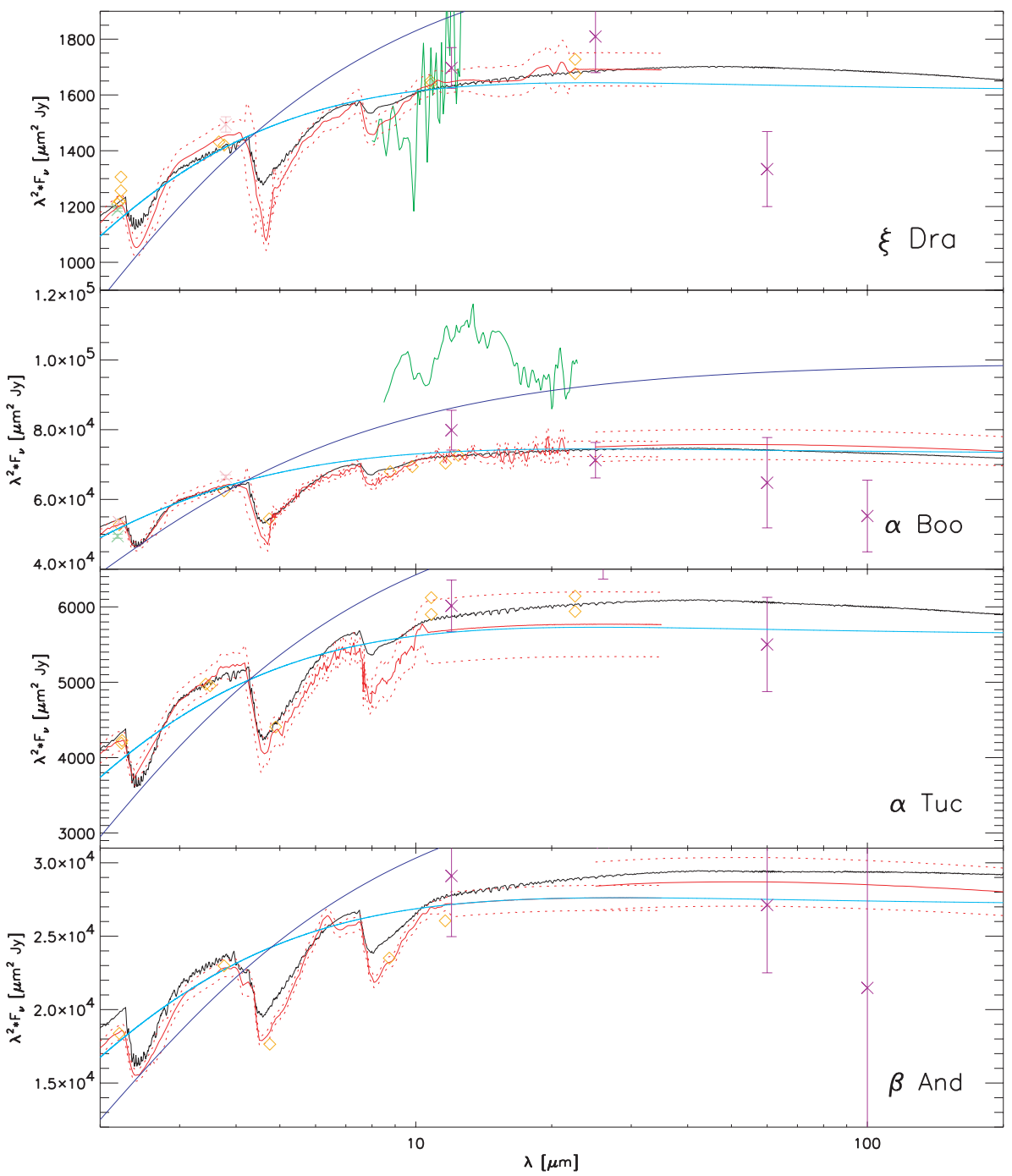

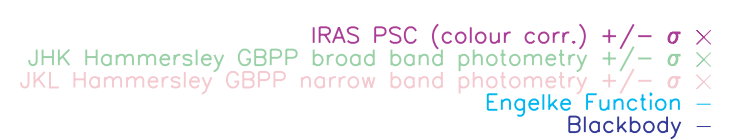

Fig. 10. Comparison between the various reference SEDs discussed in this paper in the 2 to $200 \mu \mathrm{m}$ wavelength range. $\alpha$ Boo (K2 IIIp) and $\beta$ And (M0 III) represent two composites from the CWW network, and $\xi$ Dra (K2 III) and $\alpha$ Tuc (K3 III) represent two templates. MARCS model atmosphere spectra are displayed at a resolution $\lambda / \Delta \lambda=100$. IRAS LRS and PSC data and several photometric data points collected from the ISO GBPP are also shown. instrumental resolution. In case the standard star has been properly studied using high-resolution optical and/or near-IR data and the presence of an IR excess can be excluded, one can constrain $T_{\text {eff }}$ and the temperature distribution to within $\sim 50 \mathrm{~K}$, yielding near-IR (continuum + line) medium-resolution predictions better than 1-2\%, mid-IR predictions better than $\sim 3 \%$ and far-IR predictions better than $\sim 5 \%$ for stars with earlier spectral type than M0. Each of the discussed spectral classes (early A dwarfs, solar analogs, and G9-M0 giants) has its own drawbacks. Consequently, with the aim of constraining the RSRF at a few percent level, the selected stellar standard candles should cover a wide diversity in spectral types to avoid biases to spectral features typical of different spectral classes.

\section{Comparison between different IR stellar reference SEDs}

It is instructive to compare the different IR stellar reference SEDs presented in this paper. We therefore have chosen to compare the proper blackbody, Engelke function, and model atmosphere spectrum with the spectra of two composites (as explained in Sect. 3.3) ( $\alpha$ Boo (K2 IIIp) and $\beta$ And (M0 III)) and two templates ( $\xi$ Dra (K2 III) and $\alpha$ Tuc (K3 III)) from the sample of CWW (see Fig. 10). Figure 10 also plots the IRAS LRS and PSC data and different photometric data points collected from the ISO Ground Based Preparatory Programme (GBPP) ${ }^{4}$ as obtained by Hammersley et al. (1998) and Hammersley \& Jourdain de Muizon (2003).

For the MARCS atmosphere spectra, stellar parameters as determined by Decin et al. (2003a) were used as input parameters. Main parameters for the absolute flux, $T_{\mathrm{eff}}$, and $\theta_{\mathrm{d}}$, are listed in Table 3. For the computation of the flux values of the Engelke function for both composites, $T_{\mathrm{eff}}$ and $\theta_{\mathrm{d}}$, as given in the headers by CWW, are used. Note that for $\beta$ And, the re-scaled (by CWW) angular diameter of 13.71 mas is used: based on the InfraRed Flux Method (IRFM), Blackwell et al. (1991) determined a lower angular diameter of 13.219 mas corresponding to the stellar temperature of $3839 \mathrm{~K}$. As noted in Sect. 3.2, the Engelke function needs a higher angular diameter (or $T_{\text {eff }}$ ) for

\footnotetext{
4 http://www.iso.vilspa.esa.es/users/expl_lib/ISo/ wwwcal/isoprep/gbpp/photom/
} 
Table 3. Stellar parameters used to for the calculation of (1.) the MARCS model atmosphere spectra (Cols. 2-5) and (2.) the Engelke function and blackbody (BB) (Cols. 6, 7) in Fig. 10. Effective temperature, $T_{\text {eff }}$, is given in Kelvin, gravity, $g$, in $\mathrm{cm} / \mathrm{s}^{2}$, and angular diameter, $\theta_{\mathrm{d}}$, in mas.

\begin{tabular}{lcccccc}
\hline \hline \multirow{2}{*}{ target } & \multicolumn{3}{c}{$\mathrm{M}$} & \multicolumn{2}{c}{ ARCs } & \multicolumn{2}{c}{ Engelke and BB } \\
& $T_{\text {eff }}$ & $\log g$ & {$[\mathrm{Fe} / \mathrm{H}]$} & $\theta_{\mathrm{d}}$ & $T_{\text {eff }}$ & $\theta_{\mathrm{d}}$ \\
\hline$\xi$ Dra & 4440 & 2.40 & 0.10 & 3.09 & 4440 & 3.09 \\
$\alpha$ Boo & 4320 & 1.50 & -0.50 & 20.66 & 4362 & 21.12 \\
$\alpha$ Tuc & 4300 & 1.35 & 0.00 & 5.90 & 4300 & 5.90 \\
$\beta$ And & 3880 & 0.95 & 0.00 & 13.48 & 3839 & 13.71 \\
\hline
\end{tabular}

low-gravity, low-temperature giants in order to attain the correct flux level. For both templates, same values as for the MARCS spectrum are used. The input for the blackbody calculation is the same as for the Engelke function.

Concentrating on the two composites, $\alpha$ Boo and $\beta$ And, of which a large part of the CWW spectrum consists of observational data, it is immediately clear that the Engelke function does a much better job than the blackbody function of representing the observed continuum stellar SED. The simple blackbody function can not even be used to estimate broad-band features in the RSRF. Between $\sim 23$ and $\sim 35 \mu \mathrm{m}$, the CWW spectrum of both composites is given by their Engelke function. As discussed in Figs. 1 and 2, the shape of the continuum flux differs between a proper MARCS model spectrum and the flux values given by the Engelke function, the reason being the neglect of the influence of the gravity and sphericity effects, which are important for giant stellar atmospheres. Between 20 and $200 \mu \mathrm{m}$, the maximum difference between the Engelke function used by CWW and the MARCS model atmosphere for $\alpha$ Boo is near $60 \mu \mathrm{m}$, where it attains $\sim 2.5 \%$, rising to $\sim 7.5 \%$ when the same $T_{\text {eff }}$ and $\theta_{\mathrm{d}}$ are used as for the MARCS model spectrum (Fig. 11).

The large absolute difference between the IRAS LRS spectrum of $\alpha$ Boo and both the MARCS model atmosphere spectrum and CWW composite ( 35\%, see also Van Malderen et al. 2004) remains unexplained. The LRS raw data are extracted from the Groningen IRAS database and calibrated with the LRSCAL routine in the GIPSY package. Although the IRAS LRS data were originally not meant to be absoluted calibrated, absolute calibration factors were determined by Volk \& Cohen (1989) and Cohen et al. (1992b). The same procedure was used by Cohen et al. (1996b), who however only needed a factor of 0.95 to splice the LRS data to the CGS3 data between 7.5 and $13 \mu \mathrm{m}$.

Inspecting the molecular absorption features in both templates, $\xi$ Dra and $\alpha$ Tuc, a clear difference is visible between the template spectrum (red) and the MARCS model atmospheres (black). As discussed in Decin et al. (2000) and in Sect 4.1, differences in abundance pattern (here mainly the $\mathrm{C}$ and $\mathrm{O}$ abundance), temperature, gravity, and metallicity result in substantial differences in strength in molecular absorption. In the case of $\xi$ Dra, the composite of $\alpha$ Boo was used to construct the template, the composite of $\alpha$ Hya (K2 II-III) was used for $\alpha$ Tuc. Inspecting the literature study of $\alpha$ Boo and $\xi$ Dra presented in Appendix D in Decin et al. (2003a) clearly shows that independent studies incorporating both objects in general yield an effective temperature higher by $\sim 120 \mathrm{~K}$, a logarithm of the gravity higher by $\sim 1 \mathrm{dex}$, and a metallicity $[\mathrm{Fe} / \mathrm{H}]$ higher by $\sim 0.50$ dex for $\xi$ Dra with respect to $\alpha$ Boo. Figure 12 compares the MARCS flux predictions of $\alpha$ Boo and $\xi$ Dra. Especially in the regions of molecular absorption, the (relative) difference

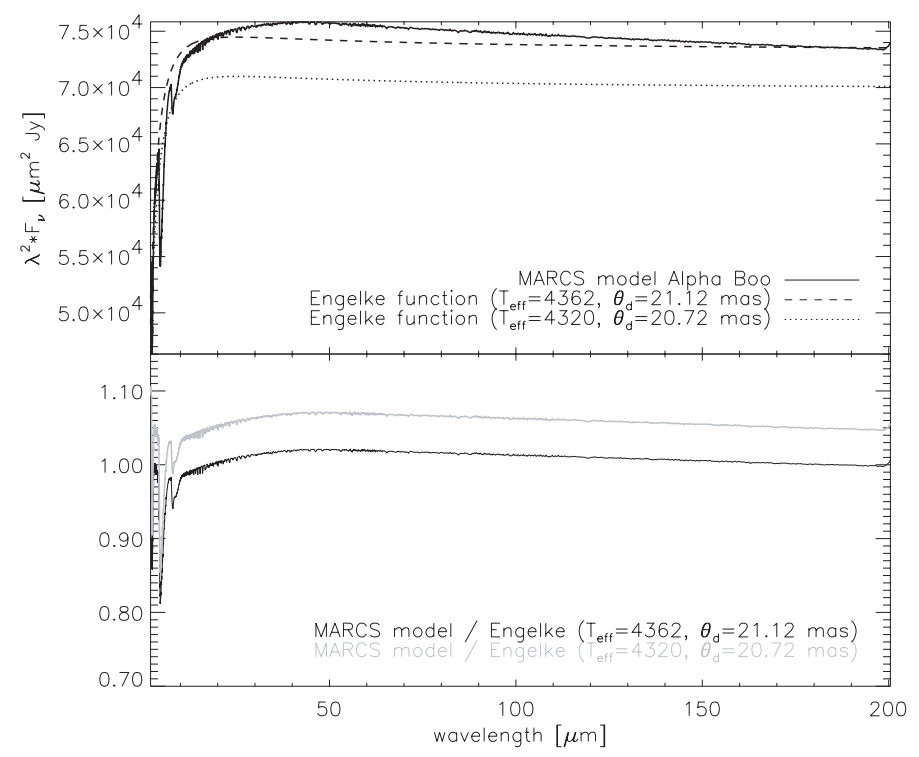

Fig. 11. Upper panel: comparison between the MARCS model spectrum of $\alpha$ Boo $(\lambda / \Delta \lambda=100)$ and predictions using the Engelke function with parameters $T_{\text {eff }}=4362 \mathrm{~K}$ and $\theta_{\mathrm{d}}=21.12$ mas (dashed line), and $T_{\text {eff }}=4320 \mathrm{~K}$ and $\theta_{\mathrm{d}}=20.72$ mas (dotted line). Bottom panel: ratio between MARCS model predictions and both Engelke functions.

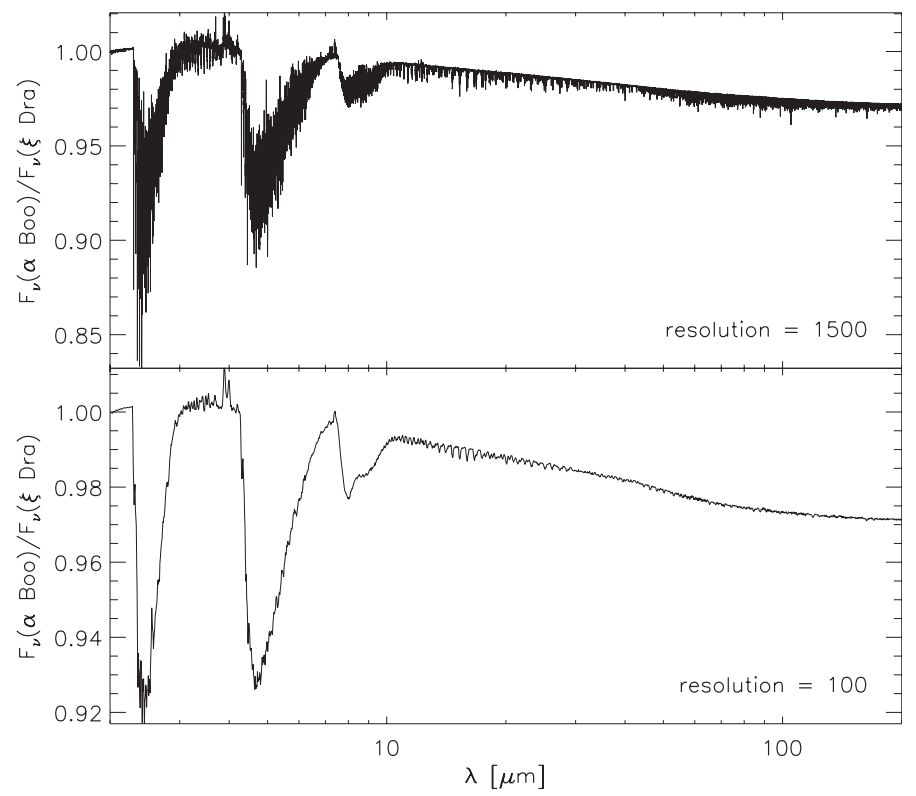

Fig. 12. Upper panel: comparison between the MARCs model spectrum of $\alpha$ Boo and $\xi$ Dra at a resolution of 1500. Both spectra have a fictitious angular diameter of 1 mas. Bottom panel: same as for the upper panel, but now at a resolution of 100. In regions of strong molecular absorption, the maximum difference is $15 \%$ for $\lambda / \Delta \lambda=1500$, and $8 \%$ for $\lambda / \Delta \lambda=100$.

between both spectra may be $10 \%$ or even larger, explaining why the difference between the CWW spectrum and the MARCS model spectrum is larger than the uncertainties quoted by CWW. Consequently, the use of the composite spectrum of the metaldeficient K2-gaint $\alpha$ Boo as a template to represent a whole class of K2 giants in the CWW network will introduce additional uncertainties, which may propagate through the RSRF determination.

For both composites, the far-IR extrapolations constructed by Cohen et al. (1996b) differ by a few percent from the MARCs 
atmospheric predictions, since the MARCS models are computed in spherical geometry, while the CWW predictions are interpolations in a grid of plane-parallel models. For the cooler, moreextended giant calibrators, sphericity effects may yield an infrared flux excess in the order of $\sim 5 \%$ (see Fig. 2).

\section{Theoretical atmosphere spectra for stellar candles and how to obtain them}

The four reference SEDs constructed from the MARCS atmosphere code presented in Fig. 10 are available via the electronic version of the article. Other reference SEDs constructed in the framework of the calibration of e.g. ISO, Spitzer, MIRI etc. are available upon request. If one needs extra reference SEDs for additional stellar calibration sources, one may always contact the authors, who are willing to provide you with the appropriate theoretical model atmosphere spectrum in the wavelength range and at the resolution requested.

\section{Conclusions}

In this paper, various sets of reference SEDs used for determining the spectrophotometric calibration of IR spectrometers (onboard satellites) have been discussed. Our main emphasis was on the stellar reference SEDs, with special focus on model atmosphere spectra. It is shown that the predicted medium-resolution IR model atmosphere spectra are accurate within 1-2\% in the near-IR, $\sim 3 \%$ in the mid-IR, and $\sim 5 \%$ in the far-IR for stars with spectral types earlier than M0. From the four types of stellar reference SEDs discussed in this study (blackbody, Engelke function, templates in the CWW network, and theoretical atmosphere spectra), it is believed that theoretical atmosphere spectra make up the best representations nowadays for the stellar SED, especially in case one wants to calibrate instruments with a spectral resolution $\gtrsim 500$. We note, however, that at $\lambda>20 \mu \mathrm{m}$, stellar calibrators might be too faint to be used in the spectrophotometric calibration pedigree.

Since the ultimate goal of the calibration system is often to be capable of deriving spectral flux values that are trustworthy to $3 \%$ or better on both absolute and relative scales, one should aim at building a highly accurate system of stellar reference SEDs. A good sky coverage by the calibrators is an important ingredient in terms of a time-efficient determination of the (spectrophotometric) calibration. In that sense, the IR network constructed by CWW is a good starting point. However, the CWW network has its limitations in terms of accuracy at representing the (molecular) spectral features and the SED at wavelengths longer than $\sim 20 \mu \mathrm{m}$. For a list of candidate calibrators, one therefore should put effort into obtaining ancillary observational data to both (1) constrain the reliability of the candidates as standard stellar sources and (2) estimate the stellar parameters to compute a set of highly reliable theoretical model atmosphere SEDs. This set should compromise standard stellar candles with different spectral types ranging between $\mathrm{A} 0 \mathrm{~V}$ and M0 III. Experiences with the Short-Wavelength Spectrometer (SWS) onboard ISO and the InfraRed Spectrometer (IRS) onboard Spitzer have shown that this set should consist of some ten to fifteen stellar calibrators. In the framework of the calibration plans for PACS onboard the ESA-Herschel satellite and MIRI onboard the NASA-JWST satellite, a set compromising theoretical spectra of $\sim 15$ stellar calibrators is in preparation (Decin et al. 2007). The input stellar parameters for each set of model spectra have to be estimated from a proper analysis of highresolution optical or near-IR data. A systematic cross-calibration with planets and asteroids is the last step in the spectrophotometric calibration pedigree. Only then can a highly accurate calibration system be developed.

Acknowledgements. L.D. acknowledges financial support from the Fund for Scientific Research - Flanders (Belgium) and KE from the Swedish Research Council. We are grateful to B. Gustafsson, B. Edvardsson, and B. Plez for their ongoing support when using the MARCS model atmosphere code developed at the Uppsala University. L.D. thanks C. Waelkens, B. Vandenbussche, T. Verhoelst, J. Blommaert, and colleagues from the ISO, Spitzer, Herschel, and MIRI calibration teams for many fruitful discussions on the use of the most appropriate SEDs to represent the spectrum of standard stars used in the spectrophotometric calibration process of these infrared instruments. T. Verhoelst is thanked for Fig. 3.

\section{References}

Absil, O., di Folco, E., Mérand, A., et al. 2006, A\&A, 452, 237

Asplund, M., Grevesse, N., Sauval, A. J., Allende Prieto, C., \& Kiselman, D. 2004, A\&A, 417, 751

Aufdenberg, J. P., Merand, A., Coude du Foresto, V., et al. 2006, ArXiv Astrophysics e-prints

Ayres, T. R., \& Linsky, J. L. 1975, ApJ, 200, 660

Ayres, T. R., \& Testerman, L. 1981, ApJ, 245, 1124

Ayres, T. R., Plymate, C., \& Keller, C. U. 2006, ApJS, 165, 618

Barber, R. J., Tennyson, J., Harris, G. J., \& Tolchenov, R. N. 2006, MNRAS, 368, 1087

Barklem, P. S., Piskunov, N., \& O’Mara, B. J. 2000, A\&A, 355, L5

Bell, K. L., \& Berrington, K. A. 1987, J. Phys. B Atom. Mol. Phys., 20, 801

Bessell, M. S., Scholz, M., \& Wood, P. R. 1996, A\&A, 307, 481

Bishop, J., Romani, P. N., \& Atreya, S. K. 1998, Plan. \& Space Sci., 46, 1

Blackwell, D. E., Lynas-Gray, A. E., \& Petford, A. D. 1991, A\&A, 245, 567

Bohlin, R. C. 2007, in The Future of Photometric, Spectrophotometric and

Polarimetric Standardization, ed. C. Sterken, ASP Conf. Ser., 364, 315

Castelli, F., \& Kurucz, R. L. 2004, ArXiv Astrophysics e-prints

Cohen, M. 2007, in The Future of Photometric, Spectrophotometric and Polarimetric Standardization, ed. C. Sterken, ASP Conf. Ser., 364, 333

Cohen, M., Walker, R. G., Barlow, M. J., \& Deacon, J. R. 1992a, AJ, 104, 1650

Cohen, M., Walker, R. G., \& Witteborn, F. C. 1992b, AJ, 104, 2030

Cohen, M., Witteborn, F. C., Walker, R. G., Bregman, J. D., \& Wooden, D. H. 1995, AJ, 110, 275

Cohen, M., Witteborn, F. C., Bregman, J. D., et al. 1996a, AJ, 112, 241

Cohen, M., Witteborn, F. C., Carbon, D. F., et al. 1996b, AJ, 112, 2274

Cohen, M., Walker, R. G., Carter, B., et al. 1999, AJ, 117, 1864

Cohen, M., Megeath, S. T., Hammersley, P. L., Martín-Luis, F., \& Stauffer, J. 2003, AJ, 125, 2645

Collet, R., Asplund, M., \& Trampedach, R. 2006, ApJ, 644, L121

Decin, L. 2000, Ph.D. Thesis, University of Leuven, Belgium

Decin, L., Waelkens, C., Eriksson, K., et al. 2000, A\&A, 364, 137

Decin, L., Vandenbussche, B., Waelkens, C., et al. 2003a, A\&A, 400, 709

Decin, L., Vandenbussche, B., Waelkens, C., et al. 2003b, A\&A, 400, 679

Decin, L., Bauwens, E., \& Blommaert, J. 2007, in ASP Conf. Ser.: The Future of Photometric, Spectrophotometric and Polarimetric Standardization, ed. C. Sterken, 443

Dehaes, S., Decin, L., Groenewegen, M., et al. 2007, A\&A, in prep.

Diaz-Miller, R. I. 2007, in ASP Conf. Ser.: The Future of Photometric, Spectrophotometric and Polarimetric Standardization, ed. C. Sterken, 81 Engelke, C. W. 1992, AJ, 104, 1248

Engelke, C. W., Price, S. D., \& Kraemer, K. E. 2006, AJ, 132, 1445

Goldman, A., Schoenfeld, W. G., Goorvitch, D., et al. 1998, J. Quant. Spectrosc. Radiat. Transfer, 59, 453

Goorvitch, D., \& Chackerian, C. 1994, ApJS, 91, 483

Gray, R. O. 2007, in The Future of Photometric, Spectrophotometric and Polarimetric Standardization, ed. C. Sterken, ASP Conf. Ser., 364, 305

Grevesse, N., Noels, A., \& Sauval, A. J. 1996, in Cosmic Abundances, ed. S. S. Holt, \& G. Sonneborn, ASP Conf. Ser., 99, 117

Griffin, M. J., \& Orton, G. S. 1993, Icarus, 105, 537

Gulliver, A. F., Hill, G., \& Adelman, S. J. 1994, ApJ, 429, L81

Gustafsson, B., Bell, R. A., Eriksson, K., \& Nordlund, ̊. 1975, A\&A, 42, 407

Gustafsson, B., Edvardsson, B., Eriksson, K., et al. 2003, in Stellar Atmosphere Modeling, ed. I. Hubeny, D. Mihalas, \& K. Werner, ASP Conf. Ser., 288, 331 Hammersley, P. L., \& Jourdain de Muizon, M. 2003, in The Calibration Legacy of the ISO Mission, ed. L. Metcalfe, A. Salama, S. B. Peschke, \& M. F. Kessler, ESA SP-481, 129 
Hammersley, P. L., Jourdain de Muizon, M., Kessler, M. F., et al. 1998, A\&AS, 128,207

Hartogh, P., Medvedev, A. S., Kuroda, T., et al. 2005, J. Geophys. Res. (Planets), 110,11008

Hauschildt, P. H., Allard, F., \& Baron, E. 1999a, ApJ, 512, 377

Hauschildt, P. H., Allard, F., Ferguson, J., Baron, E., \& Alexander, D. R. 1999b, ApJ, 525, 871

Hirata, R. \& Horaguchi, T. 1995, Atomic spectral line list (http://cdsweb.u-strasbg.fr/htbin/Cat?VI/69)

Höfner, S., Gautschy-Loidl, R., Aringer, B., \& Jørgensen, U. G. 2003, A\&A, 399,589

John, T. L. 1994, A\&A, 282, 890

Jørgensen, U. G., Jensen, P., Sørensen, G. O., \& Aringer, B. 2001, A\&A, 372, 249

Kupka, F., Piskunov, N., Ryabchikova, T. A., Stempels, H. C., \& Weiss, W. W. 1999, A\&AS, 138, 119

Kurucz, R. 1993, ATLAS9 Stellar Atmosphere Programs and $2 \mathrm{~km} \mathrm{~s}^{-1}$ grid. Kurucz CD-ROM No. 13. Cambridge, MA: Smithsonian Astrophysical Observatory

Kurucz, R. L. 1970, SAO Special Rep., 308

Kurucz, R. L. 1996, in M.A.S.S., Model Atmospheres and Spectrum Synthesis, ed. S. J. Adelman, F. Kupka, \& W. W. Weiss, ASP Conf. Ser., 108, 160

Lambert, D. L. 1994, IAU Colloq. 146: Molecules in the Stellar Environment, LNP, 428, 1

Langhoff, S. R., \& Bauschlicher, C. W. J. 1993, Chem. Phys. Lett., 211, 305

Lellouch, E., Encrenaz, T., de Graauw, T., et al. 2000, Plan. Space Sci., 48, 1393

Megessier, C. 1995, A\&A, 296, 771

Moreno, R. 1998, Ph.D. Thesis, Université de Paris VI, France

Müller, T. G., \& Lagerros, J. S. V. 1998, A\&A, 338, 340

Müller, T. G., \& Lagerros, J. S. V. 2002, A\&A, 381, 324

Müller, T. G., \& Lagerros, J. S. V. 2003, in The Calibration Legacy of the ISO Mission, ed. L. Metcalfe, A. Salama, S. B. Peschke, \& M. F. Kessler, ESA SP-481, 157

Müller, T. G., Herschel Calibration Steering Group, \& ASTRO-F Calibration Team 2005, in The Dusty and Molecular Universe: A Prelude to Herschel and ALMA, ed. A. Wilson, 471

Neugebauer, G., Soifer, B. T., Beichman, C. A., et al. 1984, Science, 224, 14
Nordlund, A. 1984, Iterative solution of radiative transfer problems with spherical symmetry using a single-ray approximation (Methods in Radiative Transfer), 211

Nowotny, W., Aringer, B., Höfner, S., Gautschy-Loidl, R., \& Windsteig, W. 2005, A\&A, 437, 273

Orton, G. S., \& Burgdorf, M. J. 2003, in ESA SP-481: The Calibration Legacy of the ISO Mission, ed. L. Metcalfe, A. Salama, S. B. Peschke, \& M. F. Kessler, 147

Partridge, H., \& Schwenke, D. W. 1997, J. Chem. Phys., 106, 4618

Piskunov, N. E., Kupka, F., Ryabchikova, T. A., Weiss, W. W., \& Jeffery, C. S. 1995, in ASP Conf. Ser. 81: Laboratory and Astronomical High Resolution Spectra, ed. A. J. Sauval, R. Blomme, \& N. Grevesse, ASP, 610

Plez, B., Brett, J. M., \& Nordlund, A. 1992, A\&A, 256, 551

Polyansky, O. L., Tennyson, J., \& Bernath, P. F. 1997, J. Mol. Spectr., 186, 213

Price, S. D., Paxson, C., Engelke, C., \& Murdock, T. L. 2004, AJ, 128, 889

Reach, W. T., Megeath, S. T., Cohen, M., et al. 2005, PASP, 117, 978

Rieke, G. H., Blaylock, M., Decin, L., et al. 2007, ApJ, submitted

Rieke, G. H., Lebofsky, M. J., \& Low, F. J. 1985, AJ, 90, 900

Rudy, D. J., Muhleman, D. O., Berge, G. L., Jakosky, B. M., \& Christensen, P. R. 1987, Icarus, 71, 159

Ryabchikova, T. A., Piskunov, N. E., Kupka, F., \& Weiss, W. W. 1997, Baltic Astron., 6, 244

Ryde, N. \& Eriksson, K. 2002, A\&A, 386, 874

Sidher, S. D., Swinyard, B. M., Griffin, M. J., et al. 2003, in The Calibration Legacy of the ISO Mission, ed. L. Metcalfe, A. Salama, S. B. Peschke, \& M. F. Kessler, ESA SP-481, 153

Stilley, J. L., \& Callaway, J. 1970, ApJ, 160, 245

van der Bliek, N. S., Gustafsson, B., \& Eriksson, K. 1996, A\&A, 309, 849

van Hoof, P. A. M. 1998, in Stellar Evolution, Stellar Explosions and Galactic Chemical Evolution, ed. A. Mezzacappa, Institute of Physics Publishing, 67

Van Malderen, R., Decin, L., Kester, D., et al. 2004, A\&A, 414, 677

Vanhollebeke, E. 2003, Master's thesis, University of Leuven, Belgium

Verhoelst, T. 2005, Ph.D. Thesis, K. U. Leuven, Belgium

Volk, K., \& Cohen, M. 1989, AJ, 98, 1918

Wiedemann, G., Ayres, T. R., Jennings, D. E., \& Saar, S. H. 1994, ApJ, 423, 806

Winters, J. M., Le Bertre, T., Jeong, K. S., Helling, C., \& Sedlmayr, E. 2000, A\&A, 361, 641 